\title{
EFFECTS OF KETOGENIC DIET ON CORNEAL KINDLING MOUSE MODEL
}

\author{
P. Andreeva-Gateva', D. Bakalov', Z. Sabit², B. Tenchov ${ }^{3}$ \\ ${ }^{1}$ Department of Pharmacology and Toxicology, Medical University - Sofia, Bulgaria \\ ${ }^{2}$ Department of Pathophysiology, Medical University - Sofia, Bulgaria \\ ${ }^{3}$ Department of Medical Physics and Biophysics \\ Medical Faculty, Medical University - Sofia, Bulgaria
}

\begin{abstract}
Aim/objective: Corneal kindling mouse test is a model of decreasing the seizure threshold after repetitive subchronical electrical stimuli. Ketogenic diet (KD) is used for the treatment of children with pharmacoresistant epilepsy since more than 100 years. Surprisingly, very few studies testing the effect of the KD in corneal kindling test were published. The aim of this study was to evaluate the effect of the KD on the seizure activity in corneal kindling mouse model. Methods: 50 adult male ICR mice (25-35 g) were randomly distributed in four groups, as follows: group 1 - standard diet (SD) treated controls $(n=10)$; group $2-K D$ treated $(n=10)$, group $3-$ kindled mice on SD treatment $(n=15)$; group $4-$ kindled mice on $K D$ treatment $(n=15)$. The diet was started at day one, one week before the start of the kindling and it continued for four weeks. At the end of the experiment, kindled mice were challenged with 6-Hz test and their behavior was assessed. Results: In kindled mice on SD the seizure latency time significantly decreased at days 14, 21 and 28. Mice on KD displayed relatively constant seizure latency during the experiment. At day 28 the duration of provoked seizures was statistically higher as compared with mice on $K D$ (median values 101 vs 2 sec, $p<0.05)$. Blood ketone levels were statistically higher $(p<0.05)$, and blood glucose level was statistically lower $(p<0.05)$ in the KD treated group, as compared with $S D$ treated mice. Conclusion: KD effectively suppressed the seizure activity in corneal kindling test. Further studies are needed for elucidating the molecular mechanisms which can explain this effect.
\end{abstract}

Key words: epilepsy, ketogenic diet, corneal kindling, mice model

Corresponding author: Pavlina Andreeva-Gateva, Department of Pharmacology and Toxicology, Medical Faculty, Medical University, 1, Georgi Sofiyski Street, 1431 Sofia, Bulgaria, e-mail: pandreeva_gateva@outlook.com, ORCID:0000-0002-3791-8654

\section{INTRODUCTION}

pilepsy is one of the most common chronic central nervous system (CNS) diseases. It is characterized by recurrent seizures that occur with various motor, sensory, autonomous, psychiatric and behavioral disorders. About 50 million people worldwide have epilepsy, and nearly $80 \%$ of epilepsy occurs in developing countries [1]. Sometimes epilepsy treatment can be as devastating as the disease itself, that is why there are many research efforts focused on finding non-pharmacological methods to ameliorate the symptoms and reduce the disease severity, especially in patients with pharmacoresistant epilepsy. One such treatment is the ketogenic $\operatorname{diet}(K D)$. 
The KD is a high fat, low carbohydrate, controlled protein diet. The first use of this form of therapy began in the early 20 's of past century when it was found that ketosis leads to seizure amelioration of the past century patients with epilepsy [3]. KD is considered a medical treatment option for pharmacoresistant epilepsy in children. Unfortunately, according to some studies the anti-epileptogenic effect of the diet is transient, and this might be due to age-related changes in brain biochemistry [13]. However, adults may also benefit from dietary treatments [24]. It is unknown what biochemical and molecular changes induced by KD are responsible for the antiepileptogenic effect, and what adverse reactions should be expected in long term usage. The use of appropriate animal models is essential [8].

Classical kindling epileptogenesis consists of repetitive electrical stimulation in the discrete brain structures such as the hippocampus, which progressively lead to post-discharge and behavioral seizures [9]. Instead, we use the corneal kindling mouse model instead, we used the corneal kindling mouse model to investigate the mechanisms of epileptogenesis. The pharmacological profile of the corneal kindling mouse is very close to that of partial epilepsy in humans and can effectively be used to identify the anticonvulsant potential of new antiepileptic treatments. The optic nerve is positioned to deliver direct electrical stimulation to the brain in a non-invasive manner [10]. The model is particularly useful for testing new treatments because it requires less experience, lower amounts of the tested substance, no stereotactic surgery or postoperative care, and the cost of the mouse and its care is lower than that of any of the hippocampal kindling rat model. Overall, these advantages make the corneal mouse model well suited to serve as a screening method for efficacy against seizure activity [9].

The aim of this study was to evaluate the effect of the $\mathrm{KD}$ on the provoked seizure activity in corneal kindling mouse model. To our knowledge there are only few studies which address the KD effects on this epilepsy model.

\section{MATERIALS AND METHODS}

\section{Experimental design}

Adult male ICR mice (25-35 g) were obtained from the Vivarium of Medical University of Sofia. They were kept at a controlled temperature $\left(23 \pm 2^{\circ} \mathrm{C}\right)$ and relative humidity (50-70\%), at 12-12 hr light-dark cycle and free access to rodent chow and water ad libitum. The use of the animal models was performed in accordance with "the Principles of Laboratory Animal Care" formulated by the National Society for Medical Research and the Guide for the Care and Use of Laboratory Animals', Washington DC, National Academy
Press. An approval N 244/25.9.2019 was received by the Bulgarian Food Safety Agency in accordance with the National Regulations.

Animals were randomly divided into four groups of different diet and treatment: group 1: standard rodent chow diet control group $(n=10)$; group 2: KD treated group ( $n=10$ ); group 3: kindling and standard diet combination $(n=15)$, group 4 : combination of kindling and KD $(n=15)$. The ketogenic diet used was KetoCAL 4:1, produced by Danone Nutricia, Germany. It is non-flavored, high-fat $4: 1$ long-chain fatty acid to carbohydrate and protein formula (Table 1). The diet was started at day one, one week before the start of the kindling and it continued for four weeks. Weekly, the body weight of the animals was measured.

Table 1. Comparison between energy contents and main nutrients in Nutricia KetoCAL 4:1 and standard rodent chow diet

\begin{tabular}{|l|c|c|}
\hline & $\begin{array}{c}\text { Nutricia } \\
\text { KetoCAL 4:1 }\end{array}$ & $\begin{array}{c}\text { Standard rodent } \\
\text { chow diet }\end{array}$ \\
\hline Nutrients & Per 100 g & Per 100 g \\
\hline Energy, kcal (kJ) & $705(2950)$ & $335(1400)$ \\
\hline Protein, g & 14.4 & 20 \\
\hline Carbohydrate.g & 8.2 & 59.4 \\
\hline Fiber, g & 5.3 & 4.8 \\
\hline$\bullet$ Soluble, g & 3.2 & 2.4 \\
\hline$\bullet$ Insoluble, g & 2.1 & 2.4 \\
\hline Fat,g & 69.2 & 4.8 \\
\hline$\bullet$ Saturated, g & 26.7 & 0.74 \\
\hline$\bullet$ Monounsaturated,g & 22.8 & 2 \\
\hline$\bullet$ Polyunsaturated, g & 19.7 & 1.77 \\
\hline
\end{tabular}

\section{Induction of kindling by electrical corneal stimulation}

All experiments were produced in the Department of Pharmacology and Toxicology, Medical University of Sofia, Bulgaria, with ECT Unit 57800 (UgoBasile, Italy). A 3-week corneal electrical kindling was applied to the experimental animals from groups 3 and 4 , begining from day 8 of the experiment until the end of the experiment at day 28 . The pharmacological profile of kindling epileptogenesis in mice is considered to be comparable to partial seizures in humans. Mice were electrically kindled twice a day with 3-second corneal stimulation with a current of $3 \mathrm{~mA}, 60 \mathrm{~Hz}$. At the end of the fourth week, mice were challenged with $6 \mathrm{~Hz}$ test for inducing psychomotor seizures with low-frequency stimuli, and duration of 3 seconds with corneal electrodes and a current of $44 \mathrm{~mA}$. The seizures were characterized by instantaneous stunning of the animal followed by a jaw clone, anterior limbs trembling, and abnormal behavior lasting at least 1 second. 


\section{Biochemical analysis}

At the end of the experiment, the mice were euthanized by a cervical dislocation. Blood was taken immediately and it was tested for glucose and ketone body levels with Xpress Statstrip Glucometer (Nova Biomedical Corporation, USA).

\section{Statistical analysis}

Data were tested for normality of distribution and equality of variance and when appropriate one way analysis of variance was applied, followed by Bonferroni post hoc test. Non-normally distributed variables were tested with Mann-Whitney $U$ test for comparison of two groups, or Kruskal-Wallis test for more than two groups comparison. Statistical significance was accepted if $p<0.05$. InVivoStat v.4 (UK) was used.

\section{RESULTS}

\section{Metabolic effects of $K D$ and kindling}

All mice were fed ad libitum and approximately $4.5 \mathrm{~g}$ chow/ day/mouse was consumed. No changes in the appetite were found in any of the groups. The energy density of the KD was twice higher than standard diet, as calculated from their composition. At day 28 , the body weight (BW) was calculated as a \% of the baseline - Fig. 1 .

The weight gain, expressed as median value, was significantly higher (113,75\% from the baseline BW) in the KD treated group (group 2) than in the control group (group 1) $(99,57 \%$ from the baseline BW). Statistically significant losing of weight was recorded in the standard diet kindled group (group 3) $(90,82 \%$ from the baseline BW)). The effect of kindling on the BW was abolished by KD treatment (group 4) $(106,67 \% \%$ from the baseline BW).

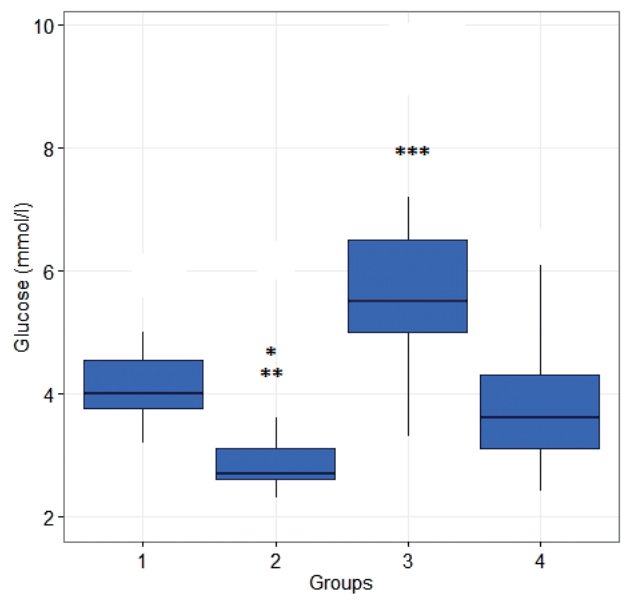

Fig. 2. Glucose levels at day 28. Data are presented as box-plot. The median is denoted by the horizontal line within the box. The box indicates the interquartile range. The whiskers extend to the most extreme data point which is no more than 1.5 times the length of the box away from the box. ${ }^{*} p<0.05$ group 2 vs group $1 .{ }^{* *} p<0.05$ group 2 vs group $3 .{ }^{* * *} p<0.05$ group 3 vs group 4
At day 28, blood glucose level was found to be significantly lower in the KD treated group (group 2, median value $2.7 \mathrm{mmol} / \mathrm{l}$ ), as compared with the control group (group 1, median value $4.0 \mathrm{mmol} / \mathrm{l}$ ) and as compared with standard diet kindling group (group 3, median value $5.0 \mathrm{mmol} / \mathrm{l}$ ). Kindled mice on standard diet (group 3) had significantly higher blood glucose level than KD treated kindled mice (group 4, median value $3.6 \mathrm{mmol} / \mathrm{l}$ ) - Fig. 2. As expected, the blood ketone levels were significantly higher in both KD treatment groups, i.e. group 2 (median value $0.4 \mathrm{mmol} / \mathrm{l}$ ) vs. control group (group 1 , median value $0.2 \mathrm{mmol} /$ ), and group 4 (median value $0.5 \mathrm{mmol} / \mathrm{l})$ vs. control group, as well as vs standard diet kindled group (group 3, median value $0.25 \mathrm{mmol} / \mathrm{l})$ - Fig. 3.

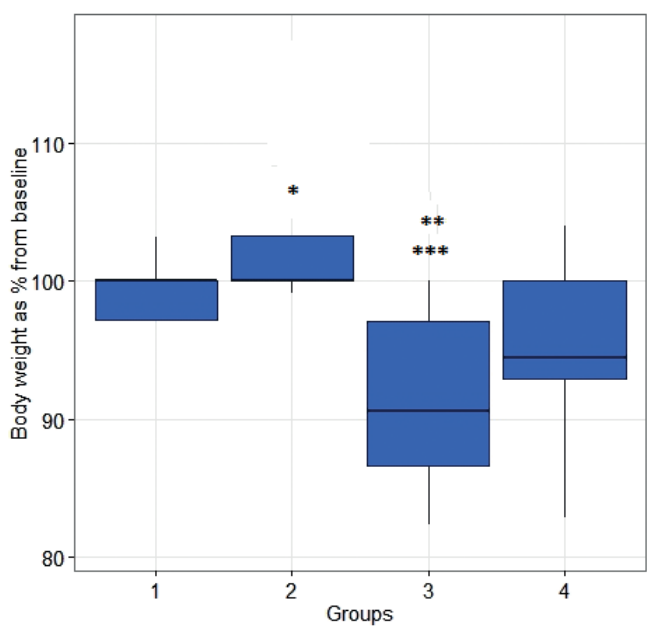

Fig. 1. Body weight at day 28 calculated as $\%$ from the baseline. Data are presented as box-plot. The median is denoted by the horizontal line within the box. The box indicates the interquartile range. The whiskers extend to the most extreme data point which is no more than 1.5 times the length of the box away from the box. ${ }^{*} p<0.05$ group 2 vs group $1 .{ }^{* *} p<0.05$ group 3 vs group 1 . ${ }^{* *} p<$ 0.05 group 3 vs group 4

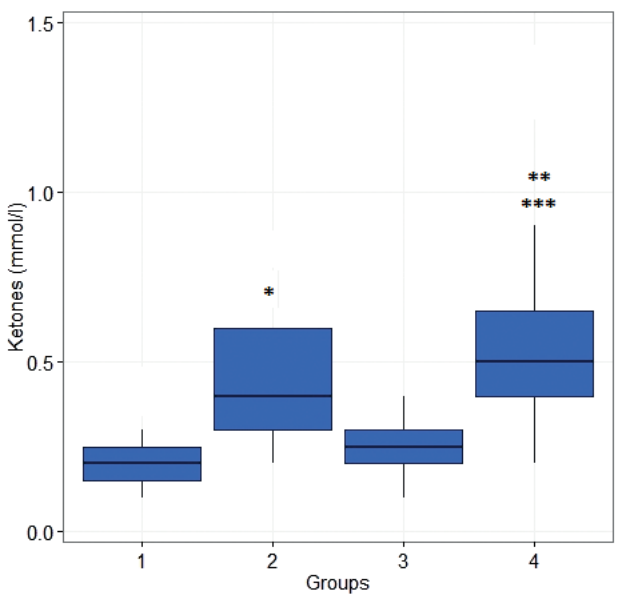

Fig. 3. Ketone levels at day 28. Data are presented as box-plot. The median is denoted by the horizontal line within the box. The box indicates the interquartile range. The whiskers extend to the most extreme data point which is no more than 1.5 times the length of the box away from the box. ${ }^{*} p<0.05$ group 2 vs group $1 .{ }^{* *} p<0.05$ group 4 vs group $1 .{ }^{* * *} p<0.05$ group 4 vs group 3 


\section{Effects of ketogenic diet on the seizure activity}

The typical behavior of the mice after kindling procedure was stunting followed by restlessness and rearing, aggressive behavior, facial clonus and head shaking. At the day $28,80 \%$ of the kindled mice displayed this type of behavior compared to the KD treated group where no more than $10 \%$ had such behavior. The time elapsed between the electrical challenge and beginning of seizure is presented in Fig. 4.

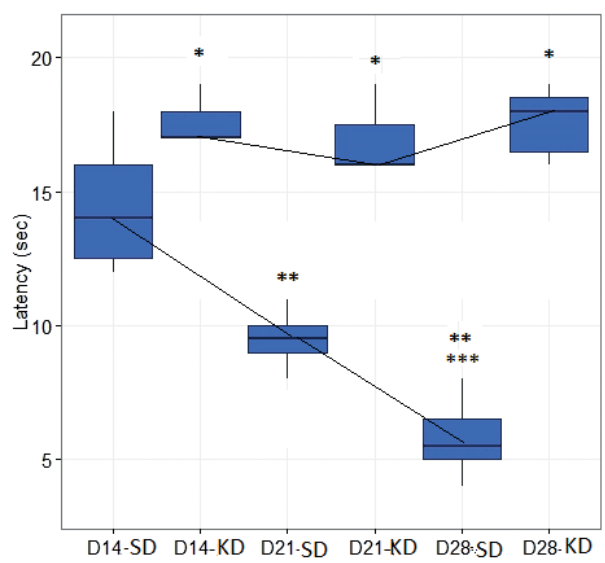

Fig. 4. Latency time between electrical challenge and seizure activity. $\mathrm{D}$ denotes the day of evaluation. SD - standard diet treated mice. KD - ketogenic diet treated mice. ${ }^{*} p<0.05$ mice on SD vs KD for corresponding days of evaluation. ${ }^{* *} p<0.05$ D21 or D28 vs $\mathrm{D} 14$ in the SD treatment group. ${ }^{* * *} p<0.05$ D28 vs D21 in the $\mathrm{SD}$ treated group

Mice treated with KD displayed statistically significantly longer latency as early as 14 days after the start of the experiment. The difference in the seizureprone activity remained statistically significant until the end of the experiment. The $6 \mathrm{~Hz}$ test applied at the end of the study demonstrated statistically longer duration of provoked seizures in the SD treated mice as compared with KD treated (median values $101 \mathrm{vs}$ 2 sec, p < 0.05) - Fig. 5 .

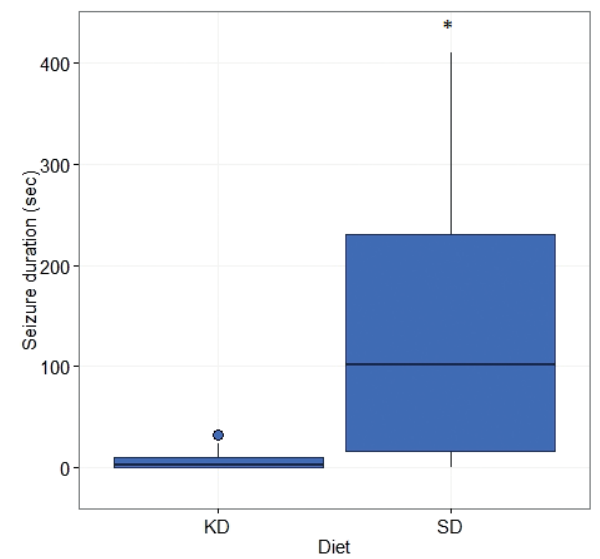

Fig. 5. Seizure durations after the $6 \mathrm{~Hz}$ test at the end of the study. $\mathrm{KD}$ - ketogenic diet. SD - standard diet. * $\mathrm{P}<0.05$

\section{DISCUSSION}

We found that KD provided significant protection in kindled mice. Our results demonstrated stable seizure latency times immediately after kindling in the group fed with KD, and a steep decrease in the same parameter in the group fed with SD. Also, there is a very significant difference between seizure duration in the treated group compared to the normal diet group.

The specific mechanism behind the beneficial effect of the KD in epilepsy is still unclear. Many different propositions were given: 1) reduction in neural excitability, 2) changes in energy availability, 3) anticonvulsive action of the ketone bodies. Some researchers suggest that KD may lead to an imbalance in CNS neurotransmitters: decreased excitatory (glutamate) and increased inhibitory (GABA), by increasing the turnover of glutamate into GABA [14]. Other researchers have suggested how KD elicits protection, but still, there is no definitive answer [15-21].

The metabolic effects of KD treatment were evident. By measuring the levels of blood ketones and glucose we found a significant increase of ketone bodies and a decrease of glucose in the treated groups, compared to both the standard diet controls and the untreated kindled group. That led us to speculate on the possibility that KD produced a protective effect by affecting brain energy metabolism, which is one of the most popular hypotheses [21, 22]. Since KD contains extremely low levels of carbohydrates, lipids are the key source of energy. The lack of carbohydrates and the abundance of fat in the diet cause activation of beta-oxidation in the liver which leads to ketone production [23]. The connection between ketosis and seizure protection was widely studied, but the findings are inconclusive [15-21].

However, as already mentioned, the original idea behind KD was inspired by a decrease in seizures during starvation. In animal models of epilepsy, there is a positive relationship between ketosis levels and seizure protection [4]. In our study, the level of ketone bodies was significantly higher in the treated group than in the standard diet mice group, but unfortunately, the results did not show a correlation between blood ketone levels and the extent of KD anti-epileptogenic effects. However, we only measured the level of ketones in blood and that is why we cannot eliminate the hypothesis that the level of ketones in the brain tissue may have a protective effect against epilepsy.

Some studies have shown that ketosis stimulates gaminobutyric acid (GABA) synthesis and decreases glutamate synthesis throughout the rat brain [15, 24]. Considering this information, the experimental model 
we used will be an excellent model for research on the effect of KD on neurotransmitter levels, as we did not alter directly the brain biochemistry of our mice with either systematic anesthesia or chemical kindling.

\section{CONCLUSION}

In this work, we described the protective effect of ketogenic diet on epileptogenesis in corneal kindling epileptic mice. This model represents an appropriate basis for further research and can be used as a convenient epilepsy model for studies of the anti-epileptogenic mechanism of ketogenic diet. The latter diet induced a level of ketosis which may explain this effect, but we should further investigate the molecular mechanisms behind it to better understand and utilize this treatment in everyday clinical practice.

Acknowledgment: The authors received financial support for the research from Medical University - Sofia (grant D-211/2018).

\section{Conflict of interest: none}

\section{REFERENCES}

1. World Health Organization, Epilepsy fact sheet, 2019. Retrieved from: https://www.who.int/news-room/fact-sheets/detail/epilepsy

2. Morimoto K., Fahnestock M., Racine R. Kindling and status epilepticus models of epilepsy: rewiring the brain, Prog. Neurobiol. 2004; 73: 1-60.

3. Wheless, J. W. History of the ketogenic diet. Epilepsia 2008; 49: 3-5.

4. Tonekaboni S. H., Mostaghimi P., Mirmiran P., et al. Efficacy of the Atkins diet as therapy for intractable epilepsy in children. Arch. Iran. Med. 2010; 13: 492-497.

5. Coppola G., D'Aniello A., Messana T., et al. Low glycemic index diet in children and young adults with refractory epilepsy: first Italian experience. Seizure 2011; 20: 526-528.

6. El-Rashidy O. F., Nassar M. F., Abdel-Hamid I. A., et al. Modified Atkins diet vs classic ketogenic formula in intractable epilepsy. Acta Neurol. Scand. 2013; 128: 402-408.

7. Puchalska P, Crawford PA. Multi-dimensional Roles of Ketone Bodies in Fuel Metabolism, Signaling, and Therapeutics. Cell Metab. 2017; 25(2): 262-284.

8. Kandratavicius L, Balista PA, Lopes-Aguiar C, et al. Animal models of epilepsy: use and limitations. Neuropsychiatr. Dis. Treat. 2014; 10: 1693-1705.
9. Löscher W. Animal Models of Seizures and Epilepsy: Past, Present, and Future Role for the Discovery of Antiseizure Drugs. Neurochem. Res. 2017; 42(7):1873-1888.

10. Rowley, N.M., White H.S. Comparative anticonvulsant efficacy in the corneal kindled mouse model of partial epilepsy: Correlation with other seizure and epilepsy models. Epilepsy Res. 2010. 92(2-3): 163-9.

11. Rogawski, M.A. Diverse mechanisms of antiepileptic drugs in the development pipeline. Epilepsy Res. 2006; 69(3): 273-94.

12. Racine, R.J. Modification of seizure activity by electrical stimulation: II. Motor seizure. Electroenceph. Clin. Neurophysiol. 1972; 32: 281-294.

13. Stafstrom CE. Dietary approaches to epilepsy treatment: old and new options on the menu. Epilepsy Curr. 2004; 4(6): 215-222.

14. Yudkoff, M., Daikhin, Y., Horyn, O., et al. Ketosis and brain handling of glutamate, glutamine, and GABA. Epilepsia 2008; 49(8): 73-75.

15. Rogawski, M. A., Löscher, W., Rho, J. M. Mechanisms of Action of Antiseizure Drugs and the Ketogenic Diet. Cold Spring Harb. Perspect. Med. 2016; 6(5): a022780.

16. Olson, C. A., Vuong, H. E., Yano, J. M., et al. The Gut Microbiota Mediates the Anti-Seizure Effects of the Ketogenic Diet. Cell 2018; 173(7): 1728-1741.e13.

17. Fan, Y., Wang, H., Liu, X., et al. Crosstalk between the Ketogenic Diet and Epilepsy: From the Perspective of Gut Microbiota. Mediat. Inflamm. 2019; 8373060.

18. Lutas, A., Yellen, G. The ketogenic diet: metabolic influences on brain excitability and epilepsy. Trends Neurosci. 2013; 36(1): 32-40.

19. McDonald, T., Cervenka, M. C. The Expanding Role of Ketogenic Diets in Adult Neurological Disorders. Brain Sci. 2018; 8(8): 148.

20. Paleologou, E., Ismayilova, N., Kinali, M. Use of the Ketogenic Diet to Treat Intractable Epilepsy in Mitochondrial Disorders. J. Clin. Med. 2017, 6(6): 56.

21. Maalouf, M., Rho, J. M., Mattson, M. P. The neuroprotective properties of calorie restriction, the ketogenic diet, and ketone bodies. Brain Res. Rev. 2009; 59(2): 293-315.

22. Kawamura, M. J., Ruskin, D. N., Masino, S. A. Metabolic Therapy for Temporal Lobe Epilepsy in a Dish: Investigating Mechanisms of Ketogenic Diet using Electrophysiological Recordings in Hippocampal Slices. Front. Mol. Neurosci. 2016; 9: 112.

23. Hutfles, L. J., Wilkins, H. M., Koppel, S. J., et al. A bioenergetics systems evaluation of ketogenic diet liver effects. Appl. Physiol. Nutr. Metab. 2017; 42(9): 955-962.

24. Hartman, A. L., Gasior, M., Vining, E. P., Rogawski, M. A. The neuropharmacology of the ketogenic diet. Pediatr. Neurol. 2007; 36(5): 281-292.

25. McDonald, T., Cervenka, M. C. Ketogenic Diets for Adults With Highly Refractory Epilepsy. Epilepsy curr. 2017; 17(6): 346-350.

Revised November, 2019 - Accepted December, 2019 